\title{
Traffic Sign Detection Using Region And Corner Feature Extraction Method
}

\author{
Hendra Maulana \\ Departement of Informatics \\ Faculty of Computer Science \\ UPN Veteran Jawa Timur \\ Surabaya, Indonesia \\ hendra.maulana.if@upnjatim.ac.id
}

\author{
Agung Mustika Riski \\ Departement of Informatics \\ Faculty of Computer Science \\ UPN Veteran Jawa Timur \\ Surabaya, Indonesia \\ agung.mustika.if@upnjatim.ac.id
}

\author{
Dhian Satria Yudha Kartika \\ Departement of Information System \\ Faculty of Computer Science \\ UPN Veteran Jawa Timur \\ Surabaya, Indonesia \\ dhian.satria@upnjatim.ac.id
}

\author{
Afina Lina Nurlaili \\ Departement of Informatics \\ Faculty of Computer Science \\ UPN Veteran Jawa Timur \\ Surabaya, Indonesia \\ afina.lina.if@upnjatim.ac.id
}

\begin{abstract}
Traffic signs are an important feature in providing safety information for drivers about road conditions. Recognition of traffic signs can reduce the burden on drivers remembering signs and improve safety. One solution that can reduce these violations is by building a system that can recognize traffic signs as reminders to motorists. The process applied to traffic sign detection is image processing. Image processing is an image processing and analysis process that involves a lot of visual perception. Traffic signs can be detected and recognized visually by using a camera as a medium for retrieving information from a traffic sign. The layout of different traffic signs can affect the identification process. Several studies related to the detection and recognition of traffic signs have been carried out before, one of the problems that arises is the difficulty in knowing the kinds of traffic signs. This study proposes a combination of region and corner point feature extraction methods. Based on the test results obtained an accuracy value of $76.2 \%$, a precision of 67.3 and a recall value of 78.6.
\end{abstract}

Keywords - traffic sign detection, image processing, region, corner points.

\section{INTRODUCTION}

Safety is the main thing for road users for order and security when driving, traffic signs are mandatory for all road users to obey. In fact, when driving there are many traffic signs that are not understood by road users, resulting in traffic violations and accidents. This can harm other people or road users by increasing the risk of accidents.

One solution that can reduce these violations is by building a system that can recognize traffic signs as reminders to motorists. Based on the Regulation of the Minister of Transportation of Indonesia No. 13 of 2014, traffic signs are one of the road equipment in the form of symbols, letters, numbers, sentences and or a combination thereof as instructions, warnings, prohibitions or orders for road users [1]. To reduce traffic violations caused by signs, a system that can recognize traffic signs is needed. Traffic signs can be detected and recognized visually by using a camera as a medium for retrieving information from a traffic sign. The layout of different traffic signs can affect the identification process.

So far, the popular driving guide used is still using satellite signal synchronization, which is usually referred to as a GPS (Global Position System) device. It can be seen that the system is not equipped with complete guidance such as detecting road signs or traffic signs. In its development it is also called Vision Based Navigation, which is a solution to this problem or also called Traffic Sign Detection [2].

Image processing is applied to this Traffic Sign Detection process. This process has the characteristics of input data and output information in the form of images. The term digital image processing is generally defined as processing a twodimensional image with a computer [3]. Digital image processing also includes all two-dimensional data where a series of real and complex numbers are represented by certain bits. The application of the distance set technique is carried out in the process of detecting the shape of objects from traffic signs. This technique aims to make the recognition of the shape of the object to be detected. This recognition is also known as Shape Recognition [4].

Several major car manufacturers have begun to create a smart car. Smart cars in the future are expected to have intelligence, but in a more sophisticated form, such as artificial intelligence technology or artificial intelligence. The detection and recognition system for traffic signs will be one of the important features of a Smart Car. When the smart car is activated on autopilot mode, it is hoped that the car will be able 
to recognize traffic signs and provide real-time navigation information to the driver.

Several studies related to the detection and recognition of traffic signs have been carried out. On T. Harsono, et al (2016) Research, the introduction process is carried out by using a matching template between the vector image of the signs entered (vector query) with all the signs in the database (vector template). The experimental results were quite good when the sampling size (segment) was $4 \times 4$, the average error percentage was $9.19 \%$ with a performance of 90.81 [5].

In Rastri Prathivi (2014) research, an augmented reality application was designed and built on a mobile device that aims to identify the logo using 3 methods, namely FAST, SURF and FLANN TREE. After implementing and testing, an average accuracy of $73 \%$ is produced for normal conditions, $69 \%$ for invariant scale conditions, $65 \%$ for 900 rotation conditions and $66 \%$ for 180 degree rotation conditions. The contribution to the research carried out is that the accuracy value of the SURF method is above $60 \%$ which consists of several taking angles, so that the accuracy value of the SURF is quite high even though it is carried out in various angles [6].

In the research of Khayeat, et al (2020), the Maximally Stable Extremal Regions (MSER) method is used to extract features from 2D images where the desired location is identified exclusively by the extreme property of the intensity function at the location. and beyond. Geometric properties and depletion operations are used to eliminate non-text locations. Multi-lingual OCR is used for multi-language understanding. The experimental results show the performance of the proposed method where traffic signs are detected in $92 \%$ of the tested images with a fairly high percentage of localization [7].

Based on Maulana (2018) research, the combination of the Maximally Stable Extremal Regions (MSER) and Harris Corner method is able to detect a character on a number plate in various conditions. The experimental results show the performance of the proposed method where the detection accuracy of the vehicle number plate location is $98.85 \%$ and the precision and recall values are 67.61 for precision and 79.66 for recall, respectively [8]. Based on previous studies, one of the problems that arises is the difficulty in knowing the kinds of traffic signs. Based on the above problems, we proposes a combination of the region (MSER) and corner point (Harris Corner) feature extraction methods. The proposed method is expected to increase the accuracy value in detecting traffic signs.

\section{METHOD}

This research is related to the approach of combining area descriptor methods and feature descriptions in detecting the location of traffic signs. The area descriptor uses the MSER method and the feature descriptor method uses the Harris Corner method. In this section, an overview of the proposed method for implementing feature extraction is presented. The proposed system combines the following sub-systems: Preprocessing, MSER Region Detection, Mark \& Mask Selected Region, Harris Corner Detection, Harris Corner Extraction, and Feature Matching. The process flow of this system can be seen in Figure 1.

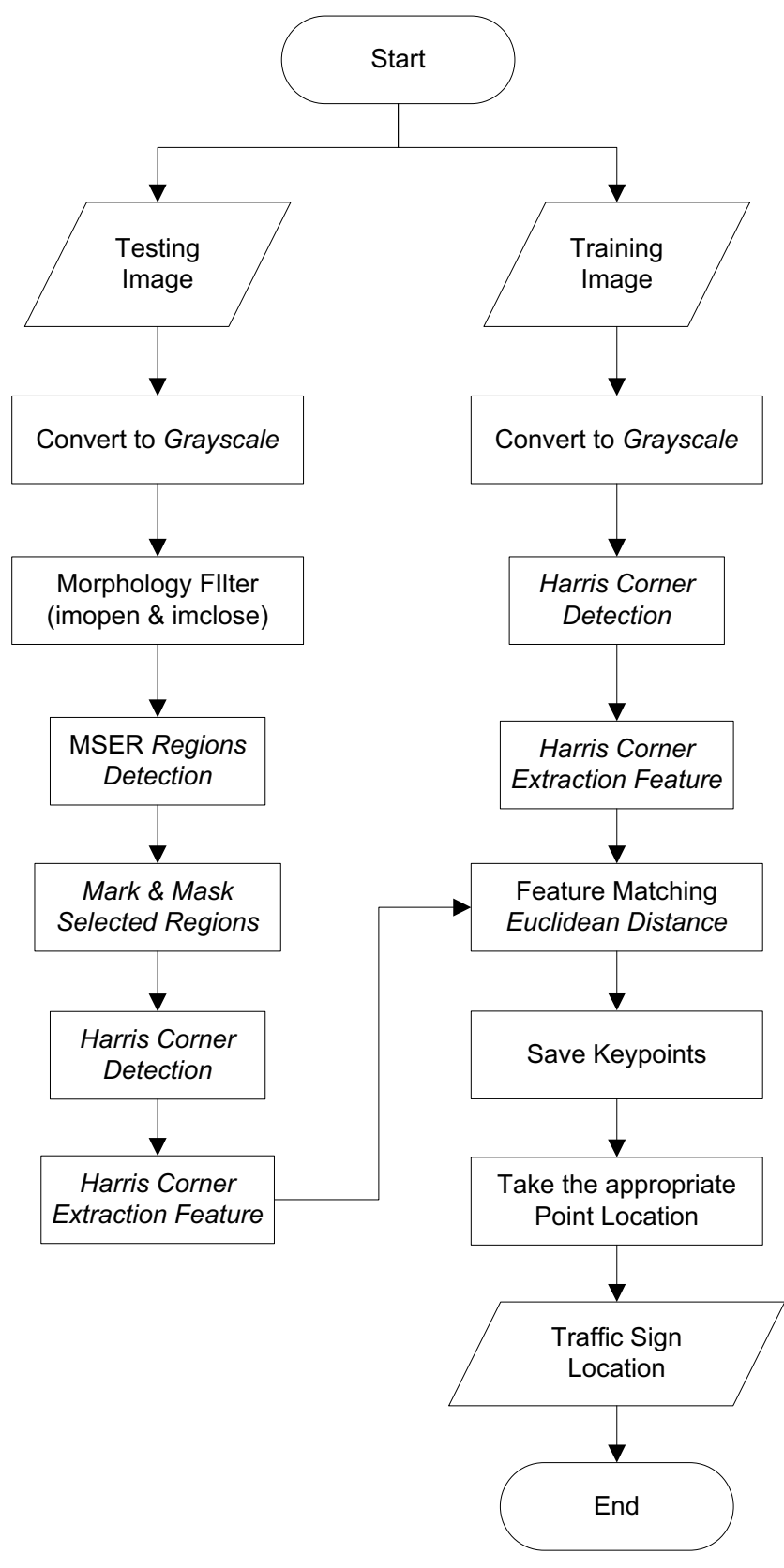

Fig. 1 Research Design

\section{A. Dataset}

The testing image dataset of 30 images is still the original image taken by the author. The training image dataset of 80 image is an image of traffic signs in Indonesia which is taken from the internet. The testing image can be seen in Figure 2 and the training image can be seen in Figure 3.
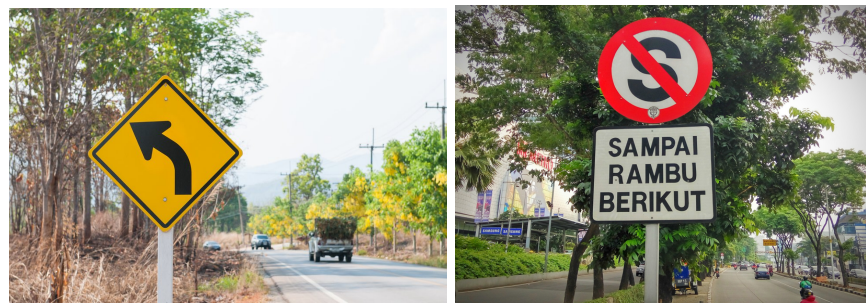

Fig. 2 Testing Image 


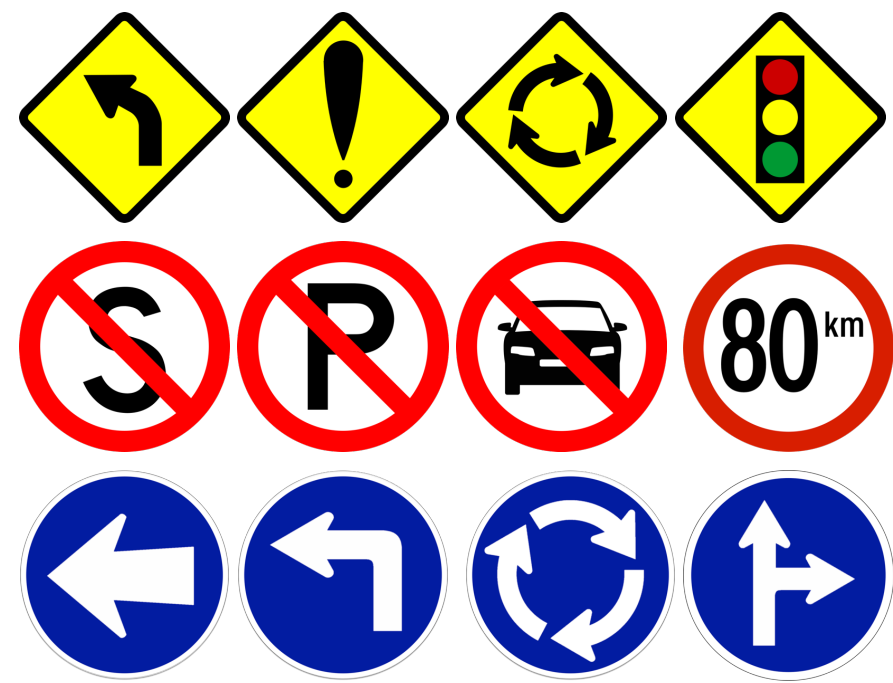

Fig. 1 Training Image

\section{B. $\quad$ Preprocessing}

The data processing process at this preprocessing stage is to correct noise in the dataset image, each test image is converted to a grayscale image, then morphological filtering is carried out using opening and closing operations to remove noise. Figure 4 shows the preprocessing stages.

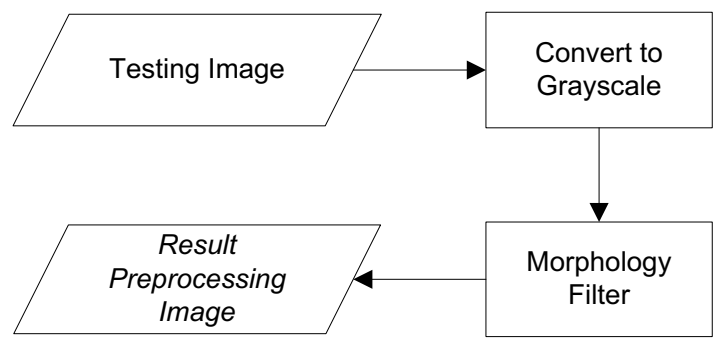

Fig. 4 Preprocessing

\section{MSER Region Detection}

The feature detection process of the MSER method is used to detect candidate traffic sign areas. The MSER feature detector works well for finding text areas (Chen, Huizhong, et al 2011). MSER detects candidate text well because consistent color and high contrast result in a stable intensity profile. The process will continue by marking the area selected by MSER, while the unselected area will be set to the default 0 (zero) which gives the image a black color. The results of this candidate detection process produce black images for areas that are not selected and grayscale images for objects selected by MSER. This process is carried out to speed up feature detection using the Harris Corner which will be carried out in the next process.

\section{Harris Corner Feature Detection}

The feature detection process of the Harris Corner method is used to detect corner points. Harris Corner Algorithm is used to extract features from the test image which will be matched with the corner point template feature.

In Corner Detection, it takes 3 derived images, namely $\mathrm{X}$ derivative, $\mathrm{Y}$ derivative, $\mathrm{X} * \mathrm{Y}$ derivative. Three convoluted images using the Prewitt operator in the previous process will be blurred with the Gaussian operator, so that a slightly blurry image will appear.
For each point in the image a $2 \times 2 \mathrm{M}$ matrix is built, and calculates the Plessey operator. Where $\mathrm{A}$ is a Blurred $\mathrm{X}^{*} \mathrm{Y}$ Derivative image, $\mathrm{B}$ is a Blurred Derivative $\mathrm{Y}$ image, and $\mathrm{C}$ is an $\mathrm{X}^{*} \mathrm{Y}$ derivative image that has been blurred from the previous Gaussian Blur process. After that, the Plessey value of each pixel can be obtained. det $(\mathrm{M})$ is the determinant of the $\mathrm{M}$ matrix, namely $(A * B)-(C * C)$ and $\operatorname{tr}(M)$ is the trace of the $\mathrm{M}$ matrix, namely $(\mathrm{A}+\mathrm{B})$.

\section{E. Testing Image Feature Extraction}

In making the testing image, the testing image of Indonesian traffic signs, keypoint detection is carried out. An example of a traffic sign template image can be seen in Figure 3. To ensure that all testing image can be used for feature matching, a keypoint search and feature extraction were carried out. The keypoint search method and feature extraction used in this process are Harris Corner. The template image with the keypoint is then used in the process of matching the features with the testing image. The Training Image feature extraction process is shown in Figure 5.

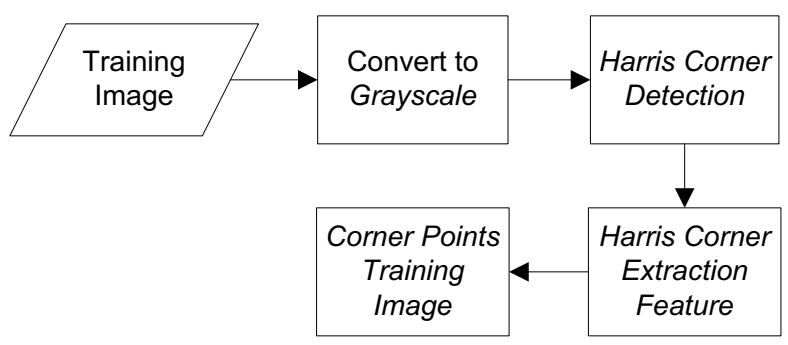

Fig. 5 Training Image Feature Extraction

\section{F. Feature Matching}

After obtaining keypoint locations, image keypoint descriptors on the training image and the test image. In the matching stage, the correlation value is calculated using the Euclidean Distance algorithm. An image can be presented as a matrix, so it can be said that an image is considered the same if each matrix cell has the same element value in the same row and column position.

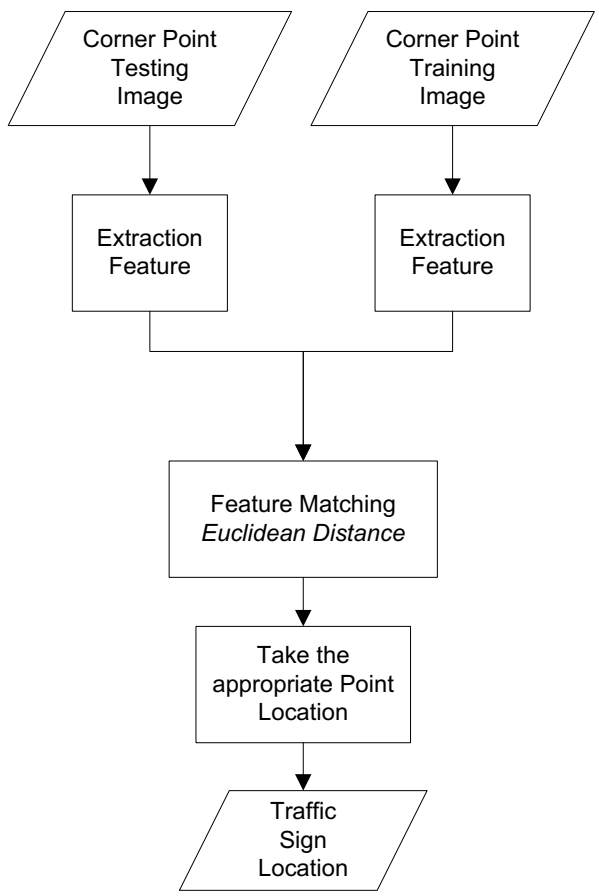

Fig. 6 Feature Maching 


\section{G. Marking of Traffic Signs Location}

After the features in the test image are identified, a suitable geometric context is used to locate the traffic sign. A bounding box will be estimated to cover the traffic sign by defining the top, bottom, left, and right dividing lines respectively. After that, we get a rough estimate of the left and right dividing lines. On the traffic sign, the ratio of the width of the sign to the height is constant. When the $\mathrm{h}$ height of the sign is estimated, we can also obtain the width of the sign. The feature matching process is shown in Figure 6.

\section{RESUlT AND DiscUSSION}

The test results and classification based on the previous explanation will be presented in more detail in this section.

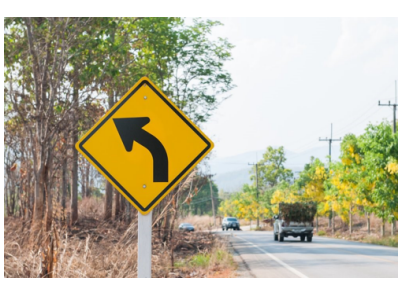

(a)

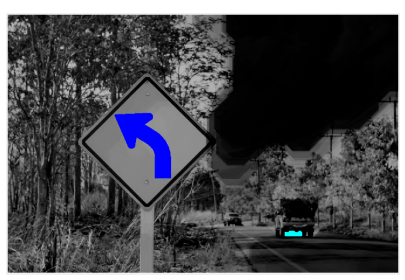

(c)

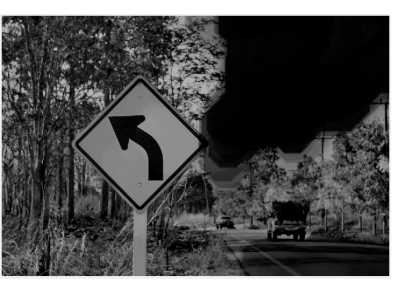

(b)

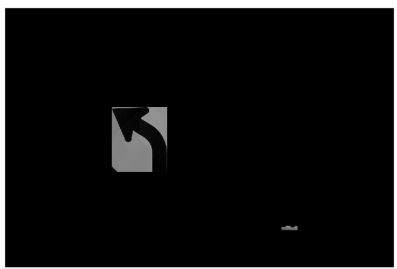

(d)

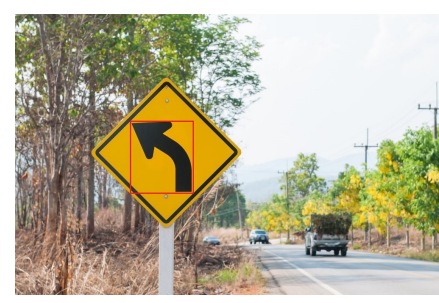

(e)

(a) testing image, (b) preprocessing, (c) MSER region, (d) feature matching result, (e) mark traffic sign location

Fig. 7 Experiment result

In the testing image, the preprocessing process will be carried out. The testing image will be converted to grayscale, then morphology filtering is carried out to remove noise using opening and closing operations. Feature extraction on MSER is done by determining the centroid of each detected region. The process will continue by marking the selected area by MSER, while the unselected area will be set by default 0 (zero) which gives a black color to the image. After obtaining the corner points from the testing image and the training image, a suitable pair of features is sought. After obtaining a suitable pair of features, the testing image is marked. Marking is done based on the corner point feature. After obtaining a suitable pair of features, the testing image is marked. Marking is done based on the corner point feature.

\section{Table 1. Result of Traffic Sign Detection}

\begin{tabular}{|c|c|c|c|}
\hline No & Accuracy & Precision & Recall \\
\hline 1 & 72.8 & 50.6 & 72.3 \\
\hline 2 & 82.3 & 70.2 & 71.1 \\
\hline 3 & 80.1 & 72.3 & 82.5 \\
\hline 4 & 50.7 & 60.9 & 55.3 \\
\hline 5 & 72.6 & 56.6 & 77.1 \\
\hline 6 & 81.8 & 74.6 & 75.2 \\
\hline 7 & 68.1 & 69.3 & 72.5 \\
\hline 8 & 53.6 & 64.3 & 58.1 \\
\hline 9 & 83.7 & 72.3 & 78.8 \\
\hline 10 & 75.1 & 70.3 & 80.5 \\
\hline 11 & 69.6 & 54.7 & 67.3 \\
\hline$\ldots$ & & & \\
\hline 30 & 80.4 & 71.4 & 74.2 \\
\hline
\end{tabular}

The results of traffic sign detection in this study can be seen in table 1.

\section{CONCLUSION}

From the observations during the process of designing, implementing, and testing software carried out, the following conclusions can be drawn:

Detection of traffic sign locations using the MSER and Harris Corner methods produces an accuracy value of $76.2 \%$, a precision of 67.3 and a recall value of 78.6. Future work will include experiments with a broader variation of images, and try combining region detector with the keypoints detector method or with other detector methods to further improve the accuracy.

\section{REFERENCE}

[1] Kementrian Perhubungan. Peraturan Menteri Perhubungan Republik Indonesia Nomor Pm 13 Tahun 2014. Kementrian Perhubungan Republik Indonesia. 2014

[2] J. Clerk Maxwell, A Treatise on Electricity and Magnetism, 3rd ed., vol. 2. Oxford: Clarendon, 1892, pp.68-73.

[3] I.S. Jacobs and C.P. Bean, "Fine particles, thin films and exchange anisotropy," in Magnetism, vol. III, G.T. Rado and H. Suhl, Eds. New York: Academic, 1963, pp. 271-350.

[4] K. Elissa, "Title of paper if known," unpublished.

[5] R. Nicole, "Title of paper with only first word capitalized," J. Name Stand. Abbrev., in press.

[6] Y. Yorozu, M. Hirano, K. Oka, and Y. Tagawa, "Electron spectroscopy studies on magneto-optical media and plastic substrate interface," IEEE Transl. J. Magn. Japan, vol. 2, pp. 740-741, August 1987 [Digests 9th Annual Conf. Magnetics Japan, p. 301, 1982].

[7] M. Young, The Technical Writer's Handbook. Mill Valley, CA: University Science, 1989. 